\title{
IL4 induces IL6-producing M2 macrophages associated to inhibition of neuroinflammation in vitro and in vivo
}

\author{
Giacomo Casella', Livia Garzetti', Alberto T. Gatta', Annamaria Finardi', Chiara Maiorino' ${ }^{1}$, Francesca Ruffini², \\ Gianvito Martino ${ }^{2}$, Luca Muzio ${ }^{2}$ and Roberto Furlan ${ }^{1 *}$
}

\begin{abstract}
Background: Myeloid cells, such as macrophages and microglia, play a crucial role in neuroinflammation and have been recently identified as a novel therapeutic target, especially for chronic forms. The general aim would be to change the phenotype of myeloid cells from pro- to anti-inflammatory, favoring their tissue-trophic and regenerative functions. Myeloid cells, however, display a number of functional phenotypes, not immediately identifiable as pro- or anti-inflammatory, and associated to ambiguous markers.

Methods: We employed in vitro assays to study macrophage polarization/differentiation in the presence of classical polarizing stimuli such as IFNY (pro-inflammatory) and IL4 (anti-inflammatory). We induced neuroinflammation in mice by immunization with a myelin antigen and treated diseased mice with intracisternal delivery of an IL4-expressing lentiviral vector. We analyzed clinical, pathological, and immunological outcomes with a focus on myeloid cells.

Results: We found that IL6, usually considered a pro-inflammatory cytokine, was released in vitro by macrophages treated with the anti-inflammatory cytokine IL4. We show the existence of macrophages expressing IL6 along with classical anti-inflammatory markers such as CD206 and demonstrate that these cells are immunosuppressive in vitro. In neuroinflamed mice, we show that IL4 delivery in the central nervous system (CNS) is associated with clinical and pathological protection from disease, associated with increased IL6 expression in infiltrating macrophages.

Conclusions: IL6 is known to mediate both pro- and anti-inflammatory effects, having two distinct ways to induce cell-signaling: either through the membrane bound receptor (anti-inflammatory) or through trans-signaling (pro-inflammatory). We show here that IL6-expressing macrophages are associated to protection from neuroinflammation, suggesting that IL6 anti-inflammatory properties prevail in the CNS, and calling for a general reconsideration of IL6 in macrophage polarization.
\end{abstract}

Keywords: IL4, IL6, M2 macrophages, Autoimmunity, EAE

\section{Background}

Plasticity and flexibility are key features of myeloid cells, like macrophages and microglia, and of their activation states. To categorize macrophage functions, currently, the M1/M2 paradigm is used [1, 2]. Generally, M1 refers to the classically activated macrophages, whereas M2 to

\footnotetext{
* Correspondence: furlan.roberto@hsr.it

${ }^{1}$ Clinical Neuroimmunology Unit, Institute of Experimental

Neurology - INSpe, Division of Neuroscience, San Raffaele Scientific Institute, Via Olgettina 58, 20132 Milan, Italy

Full list of author information is available at the end of the article
}

the alternatively activated macrophages $[3,4]$. Polarized macrophages differ in terms of receptor expression, cytokine production, effector functions, and chemokine repertoires [5]. M1 macrophages are differentiated by microbial products such as LPS, or by IFN $\gamma$ produced during an adaptive immune response by TH1 cells or during an innate immune response by natural killer (NK) cells. M1 macrophages have microbicidal or tumoricidal capacity in host defense and express, among others, iNOS, IL1 $\beta$, and TNF $\alpha$. Alternatively activated macrophages differentiate in several subtypes induced by 
various stimuli: M2a induced by IL4 and IL-13, M2b induced by exposure to immune complex (IC), and agonist of toll-like receptors (TLRs) or IL-1R, and M2c induced by IL-10 and glucocorticoids $[1,6,7]$. M2 macrophages express IL12 at low levels but display high levels of scavenging, mannose, and galactose receptors, chitinase molecules such as YM1, arginase, and chemokines such as CCL17 and CCL22. The M1/M2 paradigm is an oversimplified model to define the functional phenotypes of phagocytes, and in vivo macrophages can adopt a wide array of phenotypes depending on the stimuli coming from the tissue microenvironment [2]. Markers currently adopted to define M1/M2 macrophages, however, are ambiguous, and several of them can be found on both phenotypes. One of those is IL6, classically associated to M1 but detected also in some M2 subtypes $[1,8,9]$. IL6 is a pleiotropic cytokine, displaying both pro- and antiinflammatory activity [10]. IL6 pro-inflammatory activity descriptions prevail in literature, although its ability to promote M2 macrophage polarization has been recently reported [11]. Macrophages, along with microglia, are the main effector cells in experimental autoimmune encephalomyelitis (EAE), the mouse model for multiple sclerosis $[12,13]$. In particular, macrophage and microglia polarizations during persistent neuroinflammation are considered a crucial event for the development of chronic phases of multiple sclerosis, still lacking any treatment.

We have extensively shown the therapeutic potential of IL4 CNS gene delivery in rodent and primate models of neuroinflammation [14-19]. In previous works, we have associated the protective effect of IL4 to the modulation of $\mathrm{T}$ helper lymphocytes, and especially to the increased recruitment of $\mathrm{T}$ regulatory cells [18].

We asked the question if cells of the innate immune system are also modulated and contribute to the therapeutic effect of IL4 delivery in neuroinflammation. We show here that IL4 is able to induce IL6 release from M2 macrophages in vitro. When delivered to the CNS during neuroinflammation, IL4 modulates macrophage polarization, and we found that IL6 was specifically associated to the protective myeloid phenotype.

\section{Methods}

Bone marrow (BM)-derived macrophages in vitro culture Bone marrow (BM) cells were flushed from femur and tibia of C57BL/6 mice and cultured with macrophage colonystimulating factor, M-CSF, (100 ng ml ${ }^{-1}$, Miltenyi Biotec $\mathrm{GmbH}$, Bergisch Gladbach, Germany) in $\alpha$-minimum essential medium (MEM) (Invitrogen) for 7 days. The purity of BM-macrophage cultures was confirmed by FACS using CD11b (1:100, BD Biosciences, Mountain View, CA, USA) and F4/80 (1:100, BioLegend, San Diego, CA, USA) antibodies (average purity 80-90\%, not shown). After 7 days,
BM-derived macrophages were cultured in $\alpha$-MEM medium (Invitrogen) and differentiated to M1 and M2, respectively, with rIFN $\gamma(20 \mathrm{ng} / \mathrm{ml}$, Peprotech, USA) and rIL4 (20 ng/ml, R\&D System, Minneapolis, MN, USA) for $48 \mathrm{~h}$.

\section{Peritoneal macrophage cultures}

Sterile $3 \%$ thioglycolate (TG) was i.p. injected in C57BL/6 mice. After 5 days, mice were sacrificed, and peritoneal cells $(\mathrm{Pec})$ were recovered by lavage with saline (S.A.L.F, Bergamo, Italy). Pec were centrifuged at $1200 \mathrm{rpm}$ for $10 \mathrm{~min}$ at $4{ }^{\circ} \mathrm{C}$. Supernatants were eliminated and pellets re-suspended in RPMI 1640 medium supplemented with penicillin $(100 \mathrm{U} / \mathrm{ml})$, streptomicin $(100 \mathrm{U} / \mathrm{ml})$, and ultra-glutamine $(100 \mathrm{U} / \mathrm{ml})$ (Lonza, Milano, Italy). Cells were counted with Turk's solution (Merck Chemicals), plated at $5 \times 10^{6}$ in $60-\mathrm{mm}$ petri dishes (Becton Dickinson) and then incubated at $37{ }^{\circ} \mathrm{C}$ and $5 \% \mathrm{CO}^{2}$ for $1 \mathrm{~h}$. Finally, cells were washed twice with saline and RPMI 1640 medium supplemented with $10 \%$ FBS, penicillin $(100 \mathrm{U} / \mathrm{ml})$, streptomicin (100 U/ $\mathrm{ml})$, and ultra-glutamine $(100 \mathrm{U} / \mathrm{ml})$ was added. One hour after incubation at $37{ }^{\circ} \mathrm{C}$ and $5 \% \mathrm{CO}^{2}$, Pec polarization into $\mathrm{M} 1$ or $\mathrm{M} 2$ was performed by stimulation with $\mathrm{rIFN} \gamma(20 \mathrm{ng} / \mathrm{ml}$, Peprotech, USA) plus LPS $(100 \mathrm{ng} / \mathrm{ml})$ for $4 \mathrm{~h}$ or rIL4 $(20 \mathrm{ng} / \mathrm{ml}$, R\&D System, Minneapolis, MN, USA) for $18 \mathrm{~h}$.

\section{RT-PCR analysis}

RT-PCR analyses were performed on in vitro BM-derived macrophage assay and for IL4 gene therapy studies of the EAE model. Briefly, RNA was extracted using Trizol (Invitrogen) according to manufacturer's instructions. Residual DNA was removed by treatment with $1 \mathrm{U}$ DNase per $1 \mu \mathrm{g}$ RNA (RQ1 RNase-free DNase, Promega) at $37{ }^{\circ} \mathrm{C}$ for $30 \mathrm{~min}$. Complementary DNA (cDNA) synthesis from 3 to $5 \mu \mathrm{g}$ total RNA was performed using Ready-To-Go You-Prime First-Strand Beads (Amersham) and Random Hexamer (New England Biolabs, Ipswich, MA, USA) according to the manufacturer's instructions. Arg-1 (Mm00475988_m1), CCL17 (Mm005161 36_m1), CD206 (Mm00485148_m1), IFNY (Mm01168134_m1), IL-1 $\beta$ (Mm01336189_m1), IL4 (Mm00445259_m1), IL6 (Mm00446190_m1), iNOS (Mm00440502_m1), TNF- $\alpha$ (Mm00443258_m1), and Ym1 (Mm00657889_mH). Messenger (mRNA) levels were measured by real-time RTPCR (Applied Biosystems, Invitrogen). The 2- $\Delta \Delta C T$ method was used to calculate relative changes in gene expression [20].

\section{Fluorescence microscopy}

BM-derived and Pec macrophages, differentiated as above, were stained for CD206 FITC (R\&D) and, IL6 PE (Becton, Dickinson) for $5 \mathrm{~min}$. Cells were then fixed in PFA $4 \%$ for $10 \mathrm{~min}$ and incubated in $5 \% \mathrm{FBS}, 0.1 \%$ 
Triton X-100, and PBS for $1 \mathrm{~h}$ to block any nonspecific binding site. Nuclei were stained with DAPI. A Leica SP5 (Leica Microsystems, Milano, Italy) confocal microscope and a GE Healthcare Delta Vision were used for image acquisitions.

\section{Co-culture of $\mathrm{DCs}-\mathrm{CD} 4^{+} \mathrm{T}$ cells with $\mathrm{M} 2$ macrophages}

$\mathrm{BM}$ cells were differentiated in dendritic cells (DCs) for 6 days in RPMI complete medium with GM-CSF ( $25 \mathrm{ng} /$ $\mathrm{ml})$ and rIL4 $(25 \mathrm{ng} / \mathrm{ml})$ (R\&D Systems) and were activated with LPS $(1 \mu \mathrm{g} / \mathrm{ml})$ for $4 \mathrm{~h}$. T cells were obtained from the spleens from TCR transgenic mice (2D2) specific for the myelin oligodendrocyte glycoprotein peptide, MOG 35-55 (Espikem, Florence, Italy), on the C57Bl/6 background [21] by preparing a cell suspension by mechanical dispersion using a cell strainer, followed by positive selection of $\mathrm{CD}^{+}$cell using Miltenyi columns (Miltenyi Italy). Fifty thousand activated DCs were co-cultured with $2 \times 10^{5} \mathrm{CD}^{+} \mathrm{T}$ cells and with the following concentration of MOG peptide: $0.3,1,3,10 \mu \mathrm{M}$ in triplicates. These cultures were added $2 \times 10^{5}$ non stimulated (NS) or M2 macrophages, as indicated, and then incubated at $37^{\circ} \mathrm{C}$ and $5 \% \mathrm{CO}^{2}$ for $48 \mathrm{~h}$.

\section{ELISA assay for mIL4, IL-2, and IL6}

mIL2 and IL6 were measured from supernatants of DCs-CD4 $4^{+}$cells co-cultures with NS and M2 macrophages, by using a validated mouse-specific mouse IL2 and IL6 ELISA (R\&D Systems). mIL4 was measured in supernatants and lysates of infected cells or CSFs (withdrawn from mice cisterna magna by capillarity) from IL4-injected EAE mice, using mouse IL4 ELISA (R\&D Systems).

\section{Generation of mouse IL4-expressing lentivirus}

Murine interleukin 4 (mIL4) was obtained from a plasmid expressing mIL4 in an adenoviral vector (Ad-G/IL4) [18]. The coding sequence of mIL4 was extracted with forward and reverse primers specifically designed to contain BamHI and SalI digestion sites, both synthesized by Primm S.r.l. (Milano, Italy). A third-generation lentivirus expressing mIL4 was generated cloning the mIL4 cDNA in the backbone of a p277 lentiviral transfer vector and producing a lentivirus as previously described [22]. A GFP-expressing lentivirus was also produced to be used as negative control in all the experiments.

\section{Mice}

Six- to 8-week-old C57Bl/6 female mice were purchased from Charles River Laboratories (Calco, Italy). All mice were housed in specific pathogen-free conditions, in roomy cages, allowing free access to food and water. All efforts were made to minimize animal suffering and to reduce the number of mice used, in accordance with the
European Communities Council Directive of November 24, 1986 (86/609/EEC). All procedures involving animals were performed according to the animal protocol guidelines prescribed by the Institutional Animal Care and Use Committee (IACUC \# 449) at San Raffaele Scientific Institute (Milan, Italy). Because of the use of lentiviral vectors, animals were housed in isolated cages in the Biosafety Level 2 room of the Animal Care facility at San Raffaele Scientific Institute.

\section{IL4 gene therapy of EAE mouse model}

Chronic EAE was induced in female C57BL/6 mice, by subcutaneous with $300 \mu \mathrm{l}$ of $200 \mu \mathrm{g}$ per mouse of MOG35-55 in Freund's Adjuvant Incomplete liquid, IFA, (Sigma) supplemented with $8 \mathrm{mg} \mathrm{ml}^{-1}$ Mycobacterium tuberculosis (strain H37Ra; Difco, Lawrence, KS, USA). Pertussis toxin (500 ng, List Biological Laboratories, Campbell, CA, USA) was injected i.v. on the day of the immunization and again 2 days later. IL4-expressing lentivirus or GFP-expressing lentivirus were injected in the cisterna magna (i.c.) of the mice at 12 d.p.i. A 30-gauge needle attached to a Hamilton syringe was inserted into the intrathecal space of the cisterna magna of anesthetized mice [23]. IL4-expressing or GFP-expressing lentiviruses $(10 \mu \mathrm{l})$ in sterile phosphate-buffered saline $\left(10^{9} \mathrm{PFU} \mathrm{ml}^{-1}\right)$ were injected over $10 \mathrm{~s}$. Mice were weighed and scored for clinical signs daily up to the day of culling. Clinical assessment of EAE was performed according to the following scoring criteria: $0=$ healthy, $1=\operatorname{limp}$ tail, $2=$ ataxia and $/$ or paresis of hindlimbs, $3=$ paralysis of hindlimbs and/or paresis of forelimbs, $4=$ tetraparalysis, and $5=$ moribund or death. EAE mice were killed at 34 d.p.i for real-time PCR and histological analysis.

\section{Preparation of CNS mononuclear cells}

Mice were deeply anesthetized and perfused transcardially with cold phosphate-buffered saline at the indicated time point. The brains and the spinal cords were dissected out at the desired time point, removed, and homogenized through a $70-\mu \mathrm{m}$ cell strainer in HBSS. Mononuclear cells were isolated using a neural dissociation kit (Milteny Biotech) and by 30/37/70 \% Percoll (GE Healthcare) gradient centrifugation and collection of mononuclear cells from the $37 / 70 \%$ interphase.

\section{$\mathrm{CD}_{11 \mathrm{~b}^{+}}$cell separation}

CNS mononuclear cells were spun at $300 g$ for $10 \mathrm{~min}$ and then re-suspended in cold MACS buffer ( $1 \times$ PBS, $0.5 \%$ BSA, $2 \mathrm{mM}$ EDTA). Cells were incubated with biotinconjugated monoclonal antibodies against CD11b (Mac-1, Rat IgG2b) (Myltenyi Biotech) at a concentration of $10 \mu \mathrm{l} /$ $10^{7}$ total cells in $40 \mu \mathrm{l}$ MACS buffer for $10 \mathrm{~min}$ at $4{ }^{\circ} \mathrm{C}$. Anti-biotin microbeads $\left(20 \mu \mathrm{l} / 10^{7}\right.$ cells in $80 \mu \mathrm{l}$ MACS buffer) (Myltenyi Biotech) were added to the cells and 
incubated for $15 \mathrm{~min}$ at $4{ }^{\circ} \mathrm{C}$. Finally, the cells were loaded on the MS-columns and the column-bound CD11b ${ }^{+}$fraction isolated (Myltenyi Biotech). Cells were then centrifuged at $300 \mathrm{~g}$ for $10 \mathrm{~min}$, and re-suspended in $500 \mu \mathrm{l}$ of TRizol (Invitrogen) and frozen at $-80^{\circ} \mathrm{C}$.

\section{Histological evaluation}

At 34 d.p.i., at least three mice per group were perfused through the left cardiac ventricle with saline plus EDTA $0.5 \mathrm{mM}$ for $10 \mathrm{~min}$ followed by fixation with cold $4 \%$ paraformaldehyde, PFA, (Sigma) in $0.1 \mathrm{M}$ phosphate buffer ( $\mathrm{pH}$ 7.4). Subsequently, the spinal cords and brains from EAE mice were carefully dissected out and post-fixed in $4 \%$ PFA overnight and processed for cryogenic embedding. The quantification of neurological damage in EAE mice was performed via histological analysis of $10-\mu \mathrm{m}$ frozen CNS sections of control or IL4injected or GFP-injected EAE mice. Three different stainings were used to detect inflammatory infiltrates (hematoxylin and eosin), demyelination (Kluver Barrera), and axonal damage (Bielshowsky). Neuropathological findings were quantified on an average of 10 complete cross sections of spinal cord per mouse taken at eight different levels of the spinal cord. The number of perivascular inflammatory infiltrates were calculated and expressed as the numbers of inflammatory infiltrates per square millimeter, and demyelinated areas and axonal loss were expressed as percentage of damaged area.

\section{Immunohistochemistry and immunofluorescence}

$\mathrm{T}$ cells were stained using a rat anti-CD3 (pan-T cell marker, Cat.No. MCA1477, Serotec, Oxford, UK), CD206 positive cells were stained with rat anti-mouse CD206 (Cat.No. MCA2235, Serotec, Oxford, UK), revealed with a biotin-labeled secondary anti-rat antibody (Cat.No. BA9400, Vector Laboratories, Burlingame, CA). Macrophages were stained with biotin-labeled BS-I isolectin B4 and IB4 (Cat.No. L2140-0, Sigma-Aldrich, St. Louis, MO). Biotin-labeled antibodies were developed with the ABC kit (Cat. No. PK-6200, Vector Laboratories, Burlingame, CA) followed by liquid $\mathrm{DAB}^{+}$Substrate Chromogen System (Cat. No. K3467, Dako, Carpinteria, CA). We also stained sections with the same rat antimouse CD206 as above with a rabbit anti-mouse Iba1 (Dako, Carpinteria, CA) using fluorescent secondary antibodies as indicated. Nuclei were stained with DAPI. A Leica SP5 (Leica Microsystems, Milano, Italy) confocal microscope and a GE Healthcare Delta Vision were used for image acquisitions.

\section{Statistical analysis}

Statistical evaluations of RT-PCR data, ELISA assay, EAE score, and immunohistochemical analyses results were expressed as mean \pm s.d. or mean \pm s.e.m, as appropriate.
Results were analyzed using unpaired Student's $t$ test and Mann-Whitney $U$ test for samples with unknown and potentially disparate variances. Analyses were performed using the Prism V5.0a software (Graph-Pad, San Diego, CA, USA). Statistical significance was ranked ${ }^{*} P<0.05$, ***P<0.01, and $* * * P<0.001$.

\section{Results}

\section{IL6 is expressed by IL4-polarized macrophages}

We obtained bone marrow macrophages from C57BL/6 mice cultivated for 7 days in the presence of M-CSF. Cells where then polarized towards an M1 or M2 phenotype by using increasing concentrations of IFN $\gamma$ or IL4, respectively. The expression of classical M2 markers such as Ym1 and CCL17 (Fig. 1a, b) was indeed confined to IL4-stimulated macrophages, but also, the pro-inflammatory cytokine IL6 was selectively expressed in M2 cells (Fig. 1c). IL6 expression was induced by IL4 also in peritoneal macrophages, although at much lower levels (Additional file 1: Figure S1). Expression of classical M1 markers such as iNOS, IL-1 $\beta$, and TNF- $\alpha$ (Fig. 1d-f), were induced in IFN- $\gamma$ stimulated macrophages in a dose-dependent way. To verify if IL6 expression was indeed in the same cells bearing M2 markers and not in a subpopulation of macrophages with a different phenotype, we performed immunofluorescence on IL4-treated macrophages. We found co-expression of the M2 marker CD206 and IL6 in the same cells (Fig. 2).

\section{IL6-expressing macrophages are immunosuppressive}

We next asked if IL6-expressing M2 macrophages maintained the immunosuppressive functions associated to M2 macrophages. We stimulated T cells from 2D2 mice transgenic for the TCR specific for the myelin oligodendrocyte glycoprotein with $\mathrm{MOG}_{35-55}$, in the presence or in the absence of IL6 expressing M2 macrophages. As shown in Fig. 3, IL-2 release was significantly inhibited in the presence of IL6-expressing M2 macrophages (Fig. 3a), while IL6 was greatly increased (Fig. 3b). Thus, IL6-expressing M2 macrophages maintain their suppressive abilities.

\section{In vivo IL4 delivery to the inflamed CNS induces the expression of IL6 and M2 markers}

To verify if IL6-expressing M2 macrophages play a role also during in vivo inflammatory reactions, we induced EAE in C57BL/6 mice by immunization with $\mathrm{MOG}_{35-55}$. We injected intracisternally an IL4-expressing lentiviral vector to induce the release of IL4 in the CSF of EAE mice. Control mice were injected with a GFP-expressing lentiviral vector. We found that $\mathrm{CD}_{11 \mathrm{~b}^{+}}$myeloid cells (macrophages and microglia) purified from EAE mice treated with IL4 displayed reduced expression of the M1 

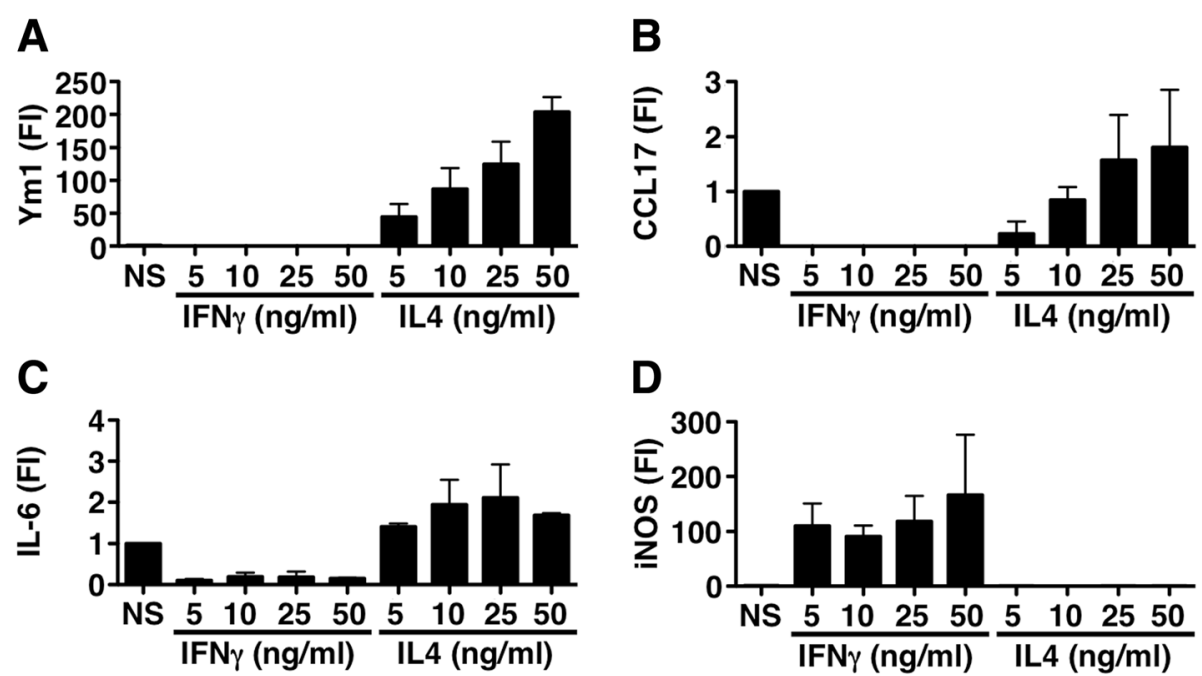

D
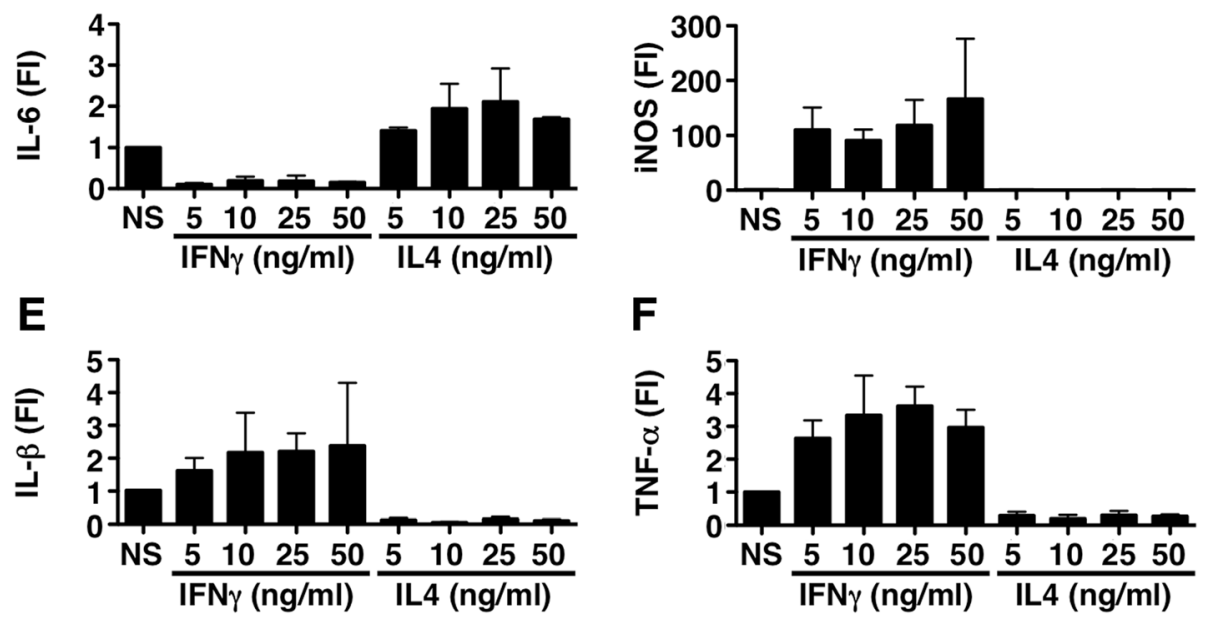

Fig. 1 Gene expression of typical M1 and M2 markers in bone marrow-derived macrophages stimulated in vitro. a-f Real-time RT-PCR for Ym1, CCL17, IL6, iNos, IL-1ß, and TNF-a, in macrophages non stimulated (NS) and stimulated with different doses of IFN- $\gamma$ (M1) and IL4 (M2). Gapdh has been used as a housekeeping gene. Data are shown as fold induction ( $\mathrm{FI} \pm$ standard deviation) over NS

marker IL-1 $\beta$, increased expression of the M2 marker Ym1, and significantly increased expression of IL6, by RT-PCR (Fig. 4a-c). Indeed, the expression of the M2 marker CD206 was significantly increased in IL4-treated EAE mice (Fig. 5a-c).
IL6-expressing IL4-treated EAE mice are protected from disease development

As previously described using different viral vectors $[14,15,18]$, IL4 gene therapy of EAE mice, after disease onset, results in a significant decrease of clinical disease
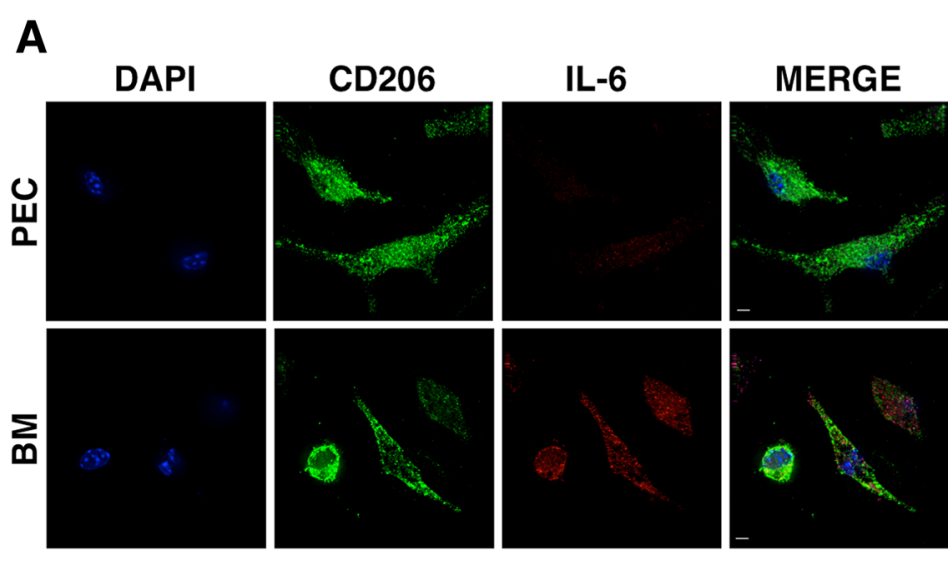

B

MERGE

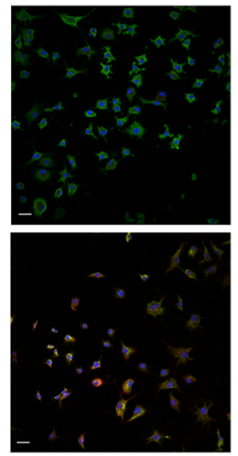

Fig. 2 rIL4 induces IL6 expression in CD206 ${ }^{+}$macrophages. a, b Immunofluorescent staining for CD206 (green) and IL6 (red) reveals co-localization of IL6 and CD206 expression in bone marrow-derived (BM) macrophages, exposed to rlL4 at $20 \mathrm{ng} / \mathrm{mL}$, but not in peritoneal (PEC) macrophages. a $\times 100$, scale bar $=10 \mu \mathrm{m}$. b $\times 40$, scale bar $=50 \mu \mathrm{m}$ 

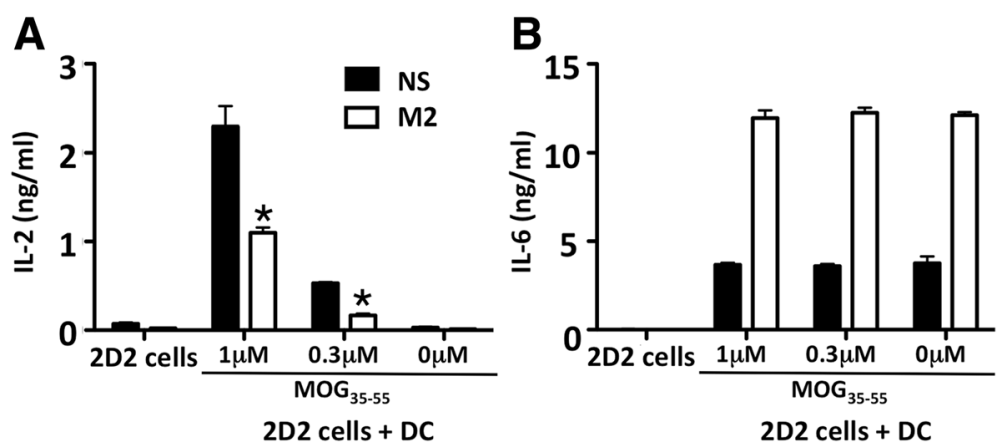

Fig. 3 IL4-stimulated macrophages releasing IL6 are immune-regulatory. IL4-stimulated bone marrow-derived macrophages (M2) reduce IL-2 release from MOG-TCR transgenic T lymphocytes (2D2) stimulated with $\mathrm{MOG}_{35-55}$ (a), as compared to non stimulated macrophages (NS), while releasing high levels of IL6 (b) as measured by ELISA

severity (Fig. 6a-d). Inhibition of EAE was confirmed also by neuropathological analysis showing a significant decrease in demyelination and axonal loss in IL4treated mice (Fig. 6g-h). We confirm here that the protective effect of IL4 gene therapy in EAE is associated to a significant increase of infiltrating $\mathrm{CD}^{+} \mathrm{T}$ cells, while the number, but not the quality, of $\mathrm{Ib} 4^{+}$myeloid cells remains unaffected (Fig. 6e, f).

\section{Discussion}

The role of innate immunity is under scrutiny in immune-mediated disorders because of its crucial role in initiating inflammation, regulating immune responses, and fostering tissue repair [2]. Investigating the role of monocytes, infiltrating and tissue-resident macrophages in immune-mediated disorders is complicated by the number of different functional phenotypes they can acquire $[1,2]$. We replicated classical in vitro polarization assays on bone marrow-derived and peritoneal macrophages. We found, surprisingly, that IL6 is associated to M2 in bone marrow-derived macrophages and in peritoneal macrophages. In in vitro polarization experiments, macrophages usually assume extreme phenotypes, probably the only condition in which the M1/M2 paradigm holds partially true. IL6 had been already described associated to different M2 phenotypes, either M2b or M2c $[1,8,9]$. This is not surprising, since several groups have reported that macrophages in vivo usually display a blend of M1 and M2 markers, hypothesizing a spectrum of possible functional phenotypes $[8,9]$. We asked the question if in our in vitro

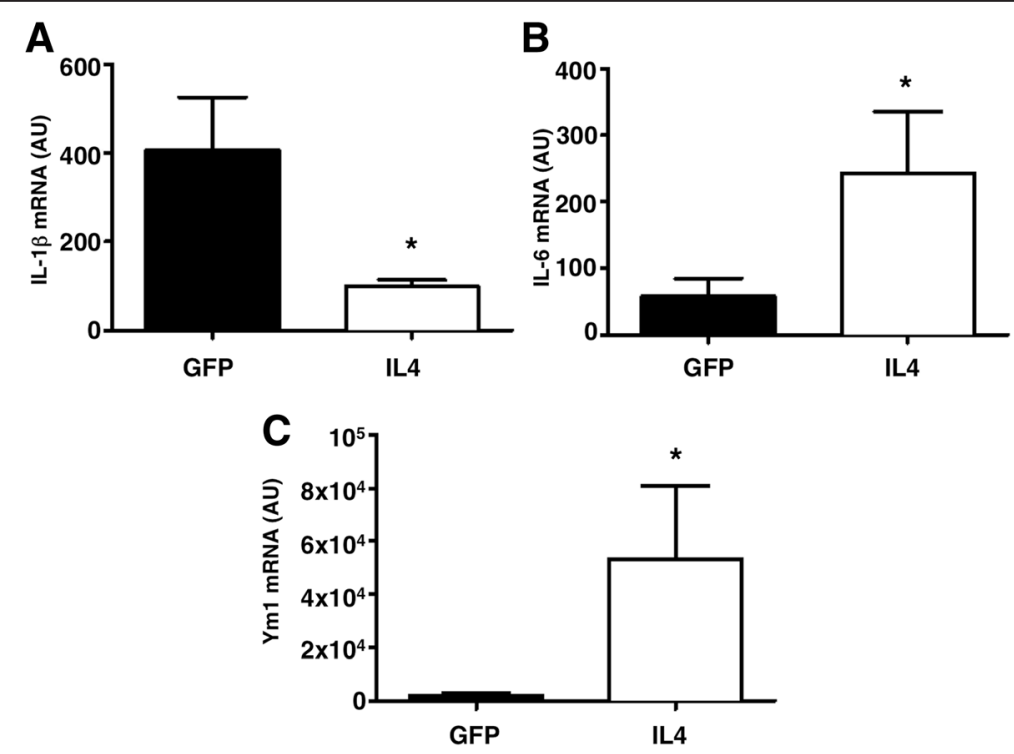

Fig. 4 IL4 gene therapy induces M2 markers and IL6 expression in CNS-infiltrating CD11 b+ cells in vivo. mRNA levels of IL6, IL-1ß, and YM1 were measured by real-time RT-PCR in CD11 b+ cells recovered from the CNS of EAE mice treated intracisternally with an IL4-expressing (IL4) or a GFP-expressing (GFP) lentivirus. IL4 gene therapy induces a decrease of IL-1ß (a), and an increase of IL6 (b) and Ym1 (c), as compared to control-treated mice. $n=3$ for each group. Gapdh has been used as a housekeeping gene. Data are shown as arbitrary units (AU \pm standard deviation). ${ }^{*} P<0.05$ ( $t$ test) 

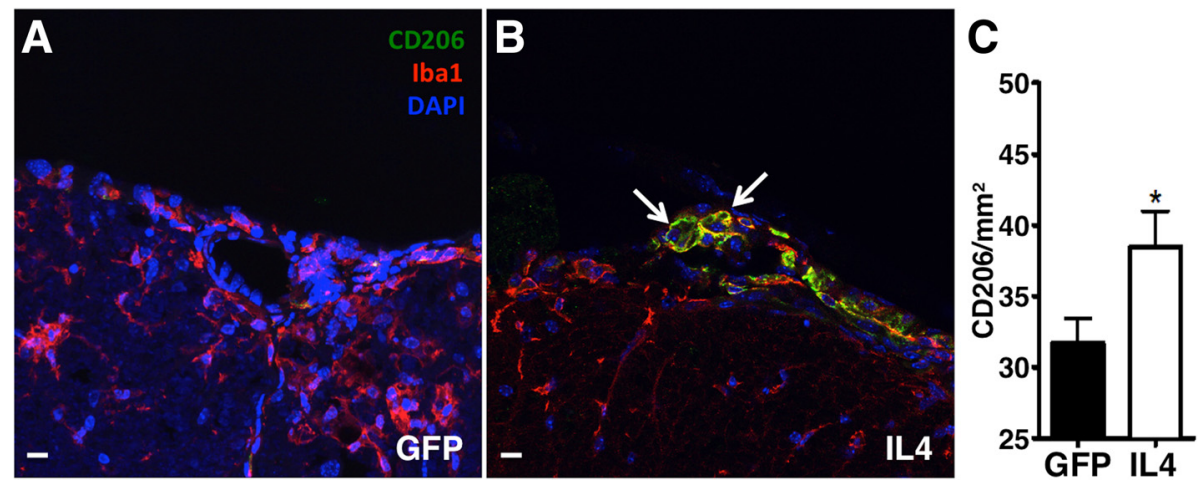

Fig. 5 IL4 gene therapy upregulates CD206 in CNS-infiltrating macrophages of EAE mice. Immunofluorescence spinal cord sections of C57BI/6 mice affected by EAE and treated, at 12 d.p.i, with the GFP-expressing (GFP, a) or the IL4-expressing lentivirus (IL4, b). The staining (CD206 in green, Iba1 in red, nuclei in blue, as indicated) highlights upregulation of CD206 in Iba ${ }^{+}$macrophages in EAE mice treated with IL4 gene therapy (b) as compared to mice treated with the GFP-expressing control virus (a). $\mathbf{a}, \mathbf{b} \times 40$, scale bar $10 \mu \mathrm{m}$. Scale quantification of CD206 ${ }^{+}$macrophages in spinal cords is shown ( \pm standard deviation) in (c). ${ }^{*} P<0.05$ ( $t$ test)

assays on bone marrow-derived macrophages, we were generating a mixture of functional phenotypes, one of them characterized by IL6 expression, or if IL6 was indeed expressed in cells bearing M2 markers. We found that IL6 was co-expressed, also at the protein level, by CD206 ${ }^{+} \mathrm{M} 2$ macrophages, and that these cells were immunosuppressive in vitro, despite releasing high levels of IL6. We have not further characterized in vitro the functional phenotype of these macrophages, namely M2a, M2b, and M2c, because of the ambiguity in the definition of these macrophage subtypes, mostly defined by the polarizing stimulus. Further, we are aware of the limitations of the in vitro suppressive assay. We therefore resolved to move to an in vivo setting, since we had already reported that induction of IL6 was associated to the anti-inflammatory effect of IL25 CNS gene therapy in a mild model of neuroinflammation [24]. We have repeatedly reported the therapeutic effect of IL4 CNS gene therapy in mouse and primate EAE [14-19]. Since IL4 is a classical M2-polarizing stimulus for macrophages, we asked the question if IL6 expression by infiltrating macrophages was associated to the protective effect of IL4 gene transfer. We found that $\mathrm{CD}_{11 \mathrm{~b}}{ }^{+}$cells purified from the brain of IL4-treated EAE mice displayed significantly increased expression of IL6, along with decreased levels of IL1 $\beta$ and increased levels of Ym1, thus a bona fide M2 phenotype, further confirmed by the increased levels of CD206 we found by immunofluorescence on infiltrating macrophages from IL4-treated EAE mice. We have currently similar findings in EAE mice successfully treated with IL27 CNS gene therapy (manuscript in preparation).

IL6 can signal through its classical receptor, a heterodimer composed by the cytokine-binding IL6R and by gp130 transducing the signal in the cell. IL6R expression is restricted to few cells in the CNS, while gp130 is ubiquitous [25]. gp130 can be activated to transduce its signal also by a complex of IL6 with the soluble form of its receptor, generated by proteolitic shedding or alternative splicing. This phenomenon, called trans-signaling, can target any gp $130^{+}$cell [26]. Outside the CNS, classical signaling is associated to IL6 protective effect, while proinflammatory activities of IL6 are mediated by transsignaling [10]. IL6 signaling in the CNS has not been extensively studied, although trans-signaling appears to be associated with pro-inflammatory activities also in the brain [27-29]. Our findings suggest that in the CNS, however, classical IL6 signaling mediating protective, anti-inflammatory, signals may outweigh trans-signaling and explain the increased IL6 release associated to the protective effects of IL4 gene therapy on neuroinflammation. These results are contradictory with a long list of papers demonstrating the detrimental effects of IL6 during neuroinflammation. Among others, IL6 knockout (k.o.) mice are resistant to EAE [30], CNS IL6 release redirects EAE inflammation to the sites of IL6 expression [31], IL6 inhibits the conversion of Th17 to Treg cells in the inflamed brain [32], and peripheral IL6 neutralization inhibits EAE [33]. It is not straightforward to reconcile these observations with our data. Most of these observations, however, rely on constitutive genetic modifications in mice, leading to possible constitutive alterations of the immune system in deletion mutants on one hand, to non physiological levels of the cytokine in IL6 overexpressing mice, on the other. In humans, monoclonal antibodies directed against IL6 are used in several chronic inflammatory diseases [10], including initial trials in neuromyelitis optica [34]. Multiple sclerosis development, however, has been reported in a patient under anti-IL6 treatment [35], leading to suspect a causal association [36]. Thus, targeting IL6 in the brain, as occurs for TNF $\alpha$ [37], may result in worsening of neuroinflammation, and suggests, rather, a prevailing protective effect of IL6 in the CNS. 

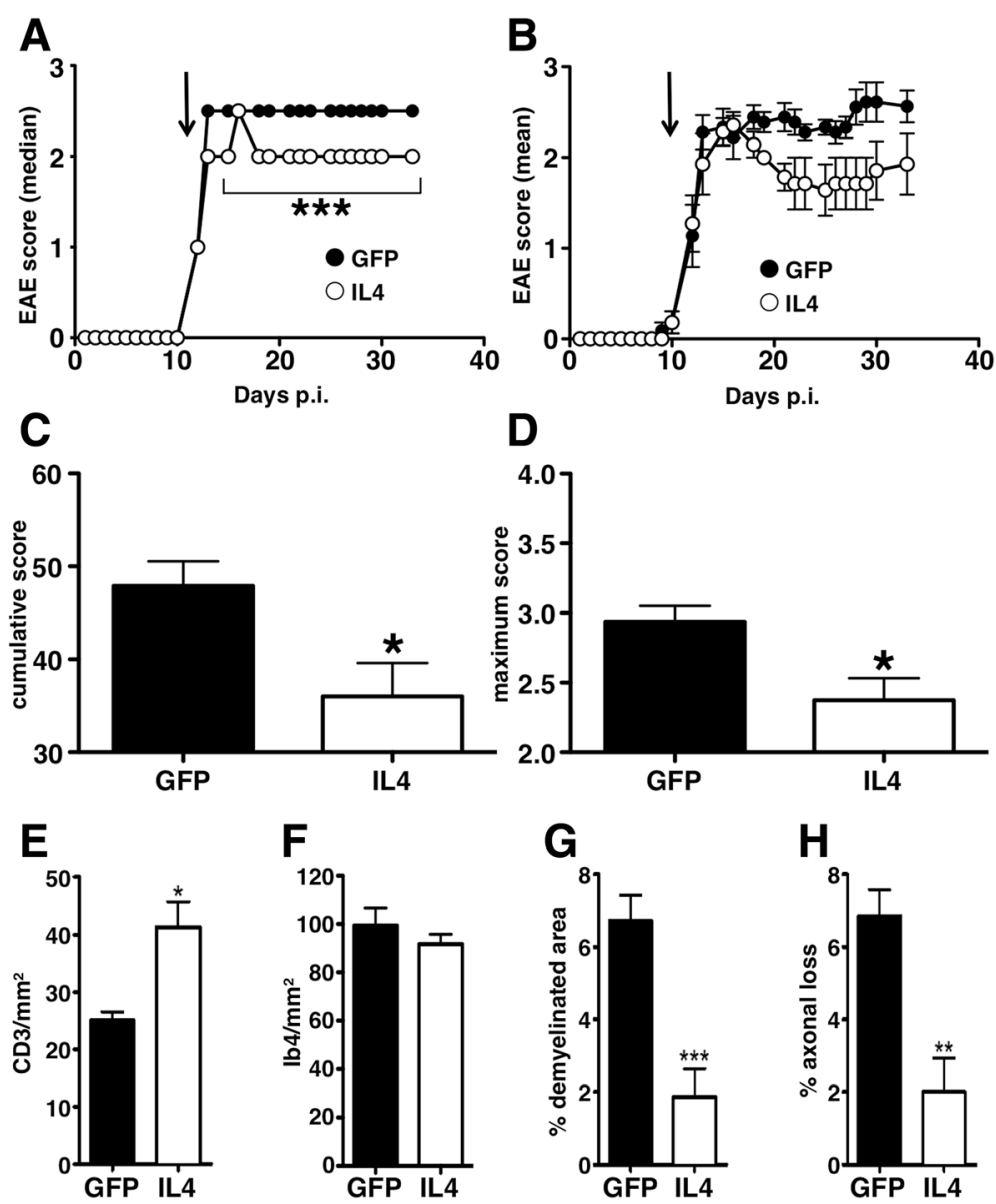

Fig. $\mathbf{6}$ CNS IL4 gene therapy inhibits clinical and pathological signs of EAE. Clinical course of EAE mice intracisternally injected with the IL4-expressing (IL4, open dots) or the GFP-expressing (GFP, closed dots) lentivirus is shown either as median (a) or mean ( \pm standard deviation) (b) values (EAE score is non-parametric). Arrows indicate the day of virus injection (day 12 post immunization). IL4-treated EAE mice are protected both in terms of clinical severity (including cumulative and maximum score, $\mathbf{a}-\mathbf{d}$ ), and demyelination $(\mathbf{g})$ and axonal loss (h). The protective effect of IL 4 gene therapy is associated to an increased number of infiltrating $\mathrm{CD}^{+} \mathrm{T}$ cells $(\mathbf{e})$, but no modulation of IB4 ${ }^{+}$myeloid cells $(\mathbf{f})$. ${ }^{* * *} P<0.0001$ (Mann-Whitney in $\mathbf{a}, t$ test in $\mathbf{g}$ ); ${ }^{*} P<0.05$ (Mann-Whitney in $\mathbf{c}, \mathbf{d}, t$ test in $\mathbf{e})$

The induction of IL6 in mice protected from EAE by IL4 gene therapy was not associated to a modulation in the number of IB4 ${ }^{+}$cells (i.e., macrophages and microglia). We confirm our previous finding that IL4 gene therapy of EAE is associated to an increase in infiltrating $\mathrm{T}$ cells that we previously described as being mostly Foxp $3^{+}[18,38]$.

\section{Conclusions}

IL6 is mostly described as a pro-inflammatory cytokine, associated to the development of several inflammatory diseases. We found that IL6 is released by bone marrowderived macrophages polarized with IL4 towards an anti-inflammatory, immune-regulatory, phenotype. Further, we found that IL6 expression was increased in infiltrating macrophages in mice protected from EAE by CNS IL4 gene delivery. We have not direct evidence of a protective role of IL6 per se; however, we think this data, together with others emerging from the literature, warrants a reconsideration of the significance of IL6 signaling during innate immune reactions in general, and during neuroinflammation in particular.

\section{Additional file}

Additional file 1: Figure S1. Gene expression of typical M1 and M2 markers in peritoneal macrophages stimulated in vitro. (A-D) real-time RT-PCR for iNOS, Ym1, CCI17, and iL6, in non stimulated (NS), and in macrophages stimulated with different doses of iFn- $\gamma(\mathrm{m} 1)$ and iL4 (M2). Gapdh has been used as a housekeeping gene. Data are shown as fold induction (Fi \pm standard deviation) over NS. (TIF 42402 kb)

\section{Abbreviations}

CNS central nervous system, EAE experimental autoimmune encephalomyelitis, MOG myelin oligodendrocyte glycoprotein, p.i post immunization, PT pertussis toxin 


\section{Acknowledgements}

L. Muzio is especially grateful to Prof. P. Brown for technical help. We are grateful to Prof. L. Naldini for providing the lentiviral backbone used.

\section{Funding}

This work has been supported by F.IS.M. Italian Foundation for Multiple Sclerosis (R.F. and G.C.) and by an unrestricted grant from EMD-Serono.

\section{Availability of data and materials}

Not applicable.

\section{Authors' contributions}

GC performed most of the EAE studies, pathological and molecular analyses, and contributed to draft the manuscript. LG performed most of the in vitro studies. ATG produced the lentiviral vectors and performed the initial EAE studies. AF designed and performed the EAE studies. CM constructed the lentiviral vector. FR supervised the immunofluorescence studies. GM and LM contributed to the study design and revised the manuscript. RF supervised the study and wrote the manuscript. All authors read and approved the final manuscript.

\section{Competing interests}

The authors declare that they have no competing interests.

\section{Consent for publication}

Not applicable.

\section{Ethics approval and consent to participate}

This experimental study was approved by our Institutional Animal Care Ethical Committee IACUC with the number \#449.

\section{Author details}

${ }^{1}$ Clinical Neuroimmunology Unit, Institute of Experimental Neurology-INSpe, Division of Neuroscience, San Raffaele Scientific Institute, Via Olgettina 58, 20132 Milan, Italy. ${ }^{2}$ Neuroimmunology Unit, Institute of Experimental Neurology_INSpe, Division of Neuroscience, San Raffaele Scientific Institute, Milan, Italy.

\section{Received: 30 March 2016 Accepted: 24 May 2016}

\section{Published online: 07 June 2016}

\section{References}

1. Biswas SK, Mantovani A. Macrophage plasticity and interaction withlymphocyte subsets: cancer as a paradigm. Nat Immunol Nature . 2010; 11:889-96. Publishing Group.

2. Italiani P, Boraschi D. From monocytes to M1/M2 macrophages: phenotypical vs functional differentiation. Front Immunol. 2013:5:514-4

3. Mantovani A, Sozzani S, Locati M, Allavena P, Sica A. Macrophage polarization: tumor-associated macrophages as a paradigm for polarized M2 mononuclear phagocytes. Trends Immunol. 2002;23:549-55.

4. Sica A, Bronte V. Altered macrophage differentiation and immune dysfunction in tumor development. J Clin Invest. 2007;117:1155-66

5. Mantovani A, Sica A, Locati M. Macrophage polarization comes of age Immunity. 2005:23:344-6.

6. Mosser DM, Edwards JP. Exploring the full spectrum of macrophage activation. Nat Rev Immunol. 2008:8:958-69.

7. Qian B-Z, Pollard JW. Macrophage diversity enhances tumor progression and metastasis. Cell. 2010;141:39-51.

8. Murray PJ, Allen JE, Biswas SK, Fisher EA, Gilroy DW, Goerdt S, et al. Perspective. Immunity Elsevier. 2014:41:14-20.

9. Bell-Temin H, Culver-Cochran AE, Chaput D, Carlson CM, Kuehl M, Burkhardt $B R$, et al. Novel molecular insights into classical and alternative activation states of microglia as revealed by stable isotope labeling by amino acids in cell culture (SILAC)-based proteomics. Mol Cell Proteomics. 2015;14:3173-84.

10. Scheller J, Garbers C, Rose-John S. Seminars in immunology. Seminars in Immunology. 2014;26:2-12. Elsevier Ltd.

11. Mauer J, Chaurasia B, Goldau J, Vogt MC, Ruud J, Nguyen KD, et al. Signaling by $\mathrm{IL}-6$ promotes alternative activation of macrophages to limit endotoxemia and obesity-associated resistance to insulin. Nat Immunol. 2014;15:423-30
12. Baeten K, Hendriks JJ, Hellings N, Theunissen E, Vanderlocht J, Ryck LD, et al. Visualisation of the kinetics of macrophage infiltration during experimental autoimmune encephalomyelitis by magnetic resonance imaging. J Neuroimmunol. 2008;195:1-6.

13. Grewal IS, Foellmer HG, Grewal KD, Wang H, Lee WP, Tumas D, et al. CD62L is required on effector cells for local interactions in the CNS to cause myelin damage in experimental allergic encephalomyelitis. Immunity. 2001;14:291-302.

14. Furlan R, Poliani PL, Galbiati F, Bergami A, GRIMALDI L, Comi G, et al. Central nervous system delivery of interleukin 4 by a nonreplicative herpes simplex type 1 viral vector ameliorates autoimmune demyelination. Hum Gene Ther. 1998;9:2605-17. Mary Ann Liebert, Inc. 2 Madison Avenue Larchmont, NY 10538 USA.

15. Furlan $\mathrm{R}$, Poliani $\mathrm{PL}$, Marconi PC, Bergami A, Ruffini F, Adorini L, et al. Central nervous system gene therapy with interleukin- 4 inhibits progression of ongoing relapsing-remitting autoimmune encephalomyelitis in Biozzi $A B / H$ mice. Gene Ther. 2001;8:13-9.

16. Poliani PL, Brok H, Furlan R, Ruffini F, Bergami A, Desina G, et al. Delivery to the central nervous system of a nonreplicative herpes simplex type 1 vector engineered with the interleukin 4 gene protects rhesus monkeys from hyperacute autoimmune encephalomyelitis. Hum Gene Ther. 2001; 12:905-20.

17. Martino G, Furlan R, Comi G, Adorini L. The ependymal route to the CNS: an emerging gene-therapy approach for MS. Trends Immunol. 2001;22:483-90. Elsevier.

18. Butti E, Bergami A, Recchia A, Brambilla E, Del Carro U, Amadio S, et al. IL4 gene delivery to the CNS recruits regulatory $T$ cells and induces clinical recovery in mouse models of multiple sclerosis. Gene Ther. 2008;15:504-15.

19. Butti E, Bergami A, Recchia A, Brambilla E, Franciotta D, Cattalini A, et al. Absence of an intrathecal immune reaction to a helper-dependent adenoviral vector delivered into the cerebrospinal fluid of non-human primates. Gene Ther. 2007;15:233-8. Nature Publishing Group.

20. Livak KJ, Schmittgen TD. Analysis of relative gene expression data using realtime quantitative $P C R$ and the $2-\Delta \Delta C T$ method. Methods. 2001;25:402-8.

21. Bettelli $E$, Pagany $M$, Weiner $H L$, Linington C, Sobel RA, Kuchroo VK. Myelin oligodendrocyte glycoprotein-specific T cell receptor transgenic mice develop spontaneous autoimmune optic neuritis. J Exp Med. 2003; 197:1073-81.

22. Follenzi A, Ailles LE, Bakovic S, Geuna M, Naldini L. Gene transfer by lentiviral vectors is limited by nuclear translocation and rescued by HIV-1 pol sequences. Nat Genet. 2000;25:217-22.

23. Furlan R, Pluchino S, Marconi PC, Martino G. Dieter Körholz and Wieland Kiess Methods in Molecular Biology ${ }^{\mathrm{TM}}$ Cytokines and Colony Stimulating Factors Methods and Protocols 10.1007/978-21. Cytokine Gene Delivery into the Central Nervous System Using Intrathecally Injected Nonreplicative Viral Vectors. Methods Mol Biol. 2003:215:279-89.

24. Maiorino C, Khorooshi R, Ruffini F, Løbner M, Bergami A, Garzetti L, et al. Lentiviral-mediated administration of IL-25 in the CNS induces alternative activation of microglia. Gene Ther. 2013;20:487-96.

25. Taga T, Kishimoto T. Gp130 and the interleukin-6 family of cytokines. Annu Rev Immunol. 1997;15:797-819.

26. Rose-John S, Neurath MF. IL-6 trans-signaling: the heat is on. Immunity. 2004;20:2-4

27. Spooren A, Kolmus K, Laureys G, Clinckers R, De Keyser J, Haegeman G, et al. Interleukin-6, a mental cytokine. Brain Res Rev. 2011;67:157-83. Elsevier B.V.

28. Rudensky AY, Campbell DJ. In vivo sites and cellular mechanisms of T reg cell-mediated suppression. J Exp Med. 2006;203:489-92.

29. Linker RA, Lühder F, Kallen K-J, Lee D-H, Engelhardt B, Rose-John S, et al. IL6 transsignalling modulates the early effector phase of EAE and targets the blood-brain barrier.

J Neuroimmunol. 2008;205:64-72.

30. Eugster HP, Frei K, Kopf M, Lassmann H, Fontana A. IL-6-deficient mice resist myelin oligodendrocyte glycoprotein-induced autoimmune encephalomyelitis. Eur J Immunol. 1998;28:2178-87.

31. Quintana A, Muller M, Frausto RF, Ramos R, Getts DR, Sanz E, et al. Sitespecific production of IL-6 in the central nervous system retargets and enhances the inflammatory response in experimental autoimmune encephalomyelitis. J Immunology. 2009;183:2079-88.

32. Korn T, Mitsdoerffer M, Croxford AL, Awasthi A, Dardalhon VA, Galileos G, et al. IL-6 controls Th17 immunity in vivo by inhibiting the conversion of conventional T cells into Foxp3+ regulatory T cells. Proc Natl Acad Sci U S A. 2008;105:18460-5. 
33. Serada S, Fujimoto M, Mihara M, Koike N, Ohsugi Y, Nomura S, et al. IL-6 blockade inhibits the induction of myelin antigen-specific Th17 cells and Th1 cells in experimental autoimmune encephalomyelitis. Proc Natl Acad Sci U S A. 2008:105:9041-6.

34. Araki M, Matsuoka T, Miyamoto K, Kusunoki S, Okamoto T, Murata M, et al. Efficacy of the anti-IL-6 receptor antibody tocilizumab in neuromyelitis optica: a pilot study. Neurology. 2014;82:1302-6. Epub 2014 Mar 14. Available from: http://eutils.ncbi.nlm.nih.gov/entrez/eutils/elink. fcgi?dbfrom=pubmed\&id=24634453\&retmode=ref\&cmd=prlinks.

35. Beauchemin P, Carruthers R. MS arising during Tocilizumab therapy for rheumatoid arthritis. Mult Scler. 2016;22:254-6.

36. Comabella M. Tocilizumab and multiple sclerosis: a causal relationship? Clinical Commentary on the case report entitled - MS arising during Tocilizumab therapy for rheumatoid arthritis. Mult Scler. 2016;22:257-8.

37. Probert L. TNF and its receptors in the CNS: the essential, the desirable and the deleterious effects. Neuroscience. 2015;302:2-22.

38. Esposito M, Ruffini F, Bergami A, Garzetti L, Borsellino G, Battistini $L$, et al. IL-17- and IFN- $\gamma$-secreting Foxp3+ T cells infiltrate the target tissue in experimental autoimmunity. J Immunol. 2010;185:7467-73. American Association of Immunologists.

\section{Submit your next manuscript to BioMed Central} and we will help you at every step:

- We accept pre-submission inquiries

- Our selector tool helps you to find the most relevant journal

- We provide round the clock customer support

- Convenient online submission

- Thorough peer review

- Inclusion in PubMed and all major indexing services

- Maximum visibility for your research

Submit your manuscript at www.biomedcentral.com/submit 